\title{
Extended Two-Particle Green's Functions and Optical Potentials for Two-Particle Scattering by Many-Body Targets
}

\author{
Joachim Brand and Lorenz S. Cederbaum
}

Theoretische Chemie, Universität Heidelberg, Im Neuenheimer Feld 253, D-69120 Heidelberg, Germany

Received May 15, 1996

\begin{abstract}
An extension of the fermionic particle-particle propagator is presented that possesses similar algebraic properties to the single-particle Green's function. In particular, this extended two-particle Green's function satisfies Dyson's equation and its self energy has the same analytic structure as the the self energy of the single-particle Green's function. For the case of a system interacting with one-particle potentials only, the two-particle self energy takes on a particularly simple form, just like the common self energy does. The new two-particle self energy also serves as a well behaved optical potential for the elastic scattering of a twoparticle projectile by a many-body target. Due to its analytic structure, the two-particle self energy avoids divergences that appear with effective potentials derived by other means.

(C) 1996 Academic Press, Inc.
\end{abstract}

\section{INTRODUCTION}

Many-body Green's functions, also called propagators, provide useful tools for investigating various properties of many-body systems like solids, atomic nuclei, atoms, and molecules. The so called single-particle Green's function [1] is the most fundamental in a whole hierarchy of related propagators. One of the reasons for the outstanding importance of the single-particle Green's function is that it satisfies a well-known equation - the Dyson equation [1]. Among the wealth of applications of this equation we want to mention that the Dyson equation has been exploited for calculations of ionisation spectra of molecules [2-6] as well as scattering cross sections [7]. In fact, the Dyson equation allows one to derive exact effective oneparticle equations for the elastic scattering of a particle by a many-body target $[8,9]$. In such a one-particle equation, a nonlocal and in general energy-dependent potential — called optical potential -includes the correlations of the many-particle system. The Green's function optical potential-also called self energy-has several advantages upon the optical potentials derived by other means, e.g. by Feshbach projection $[10,11]$ from the multiple-particle Schrödinger equation. One point is the good-natured energy dependence that avoids divergences for high energies. Also the self energy closely resembles the properties of the phenomenological optical model potentials that have been common in nuclear physics for a long time [12]. 
Further we want to mention that the self energy of a system interacting with oneparticle potentials takes on a very simple appearance and provides an exact optical potential already in first order perturbation theory. This is usually not the case for optical potentials derived by other means. In general, the well developed approximation schemes for the self energy based on perturbation theory [13] account for a balanced treatment of the correlation, both in the target and the scattering state. This balanced treatment is much more difficult to achieve in wavefunction approaches [7].

The next member in the hierarchy of Green's functions is the two-particle Green's function [1]. This is a function of three energy variables. Although this function satisfies an integral equation with kernels that can be approximated in a diagrammatic approach - the Bethe-Salpeter equation-for most problems it is too complicated to handle. Instead, one uses propagators with only one energy variable left, e.g. the polarization propagator $[1,14]$ and the particle-particle propagator [15] which have been used to calculate excitation energies $[16,17]$ and double-ionisation spectra [18-20], respectively. Furthermore, approaches have been reported starting from the latter propagator to calculate amplitudes for two particle scattering via optical potentials $[21,22]$. Although there are some analogies between these propagators and the single-particle Green's function, the Dyson equation is not passed on to these two propagators. Neither by diagrammatical analysis nor from the algebraic point of view is there any close analogon to this fundamental equation.

The main goal of this paper is to show that it is possible to construct extensions of these two-particle propagators (only the particle-particle propagator for fermions is considered explicitly) that have the major algebraic properties in common with the single-particle Green's function, namely those that lead to a Dyson equation and a two-particle self energy. It is particularly interesting to note that this twoparticle self energy serves as an optical potential for the elastic scattering of a twofermion projectile by a correlated target. Possible applications include, e.g., the scattering of a deuteron by an atomic nucleus or the scattering of a positronium by a molecule. In general, the usefulness and quality of possible approximations are determined by the algebraic properties of the optical potential. The two outstanding features of our new, two-particle self energy are the good-natured energy dependence that has a finite limit for high energies and the fact that influences resulting from a one-particle potential are accounted for exactly already in the energy-independent first order. These properties are straightforward consequences of the algebraic analogies to the single-particle Green's function, but they are in no way a matter of course with two-body effective potentials.

This paper is organized as follows: After briefly reviewing the algebraic properties of the single-particle Green's function we will show in Section II by explicit construction, that an extension of the particle-particle propagator is possible that inherits the major algebraic properties from the single-particle Green's function and satisfies a Dyson equation. Discussing the properties of the extended propagator and its self energy we also consider the special case of a system interacting only 
with one-particle potentials. In Section III we then show how the extended propagator can be used for deriving well behaved effective two-particle scattering equations. We will, however, develop these ideas in a general framework allowing for either bound or free two-particle projectile states. Also, we will state explicitly the properties of the extended two-particle propagator that are prerequisites for derivation of effective scattering equations.

\section{CONSTRUCTION OF TWO-PARTICLE PROPAGATORS}

In this section we will construct an extended two-particle propagator that inherits a couple of fundamental properties from the single-particle Green's function. Although the single-particle Green's function and the Dyson equation are well known and described thoroughly in many standard textbooks [1, 15], we briefly review the algebraic derivation of the Dyson equation [23], in order to motivate our construction of extended two-particle propagators. We carry on by applying the formalism developed for the single-particle Green's function to the particleparticle propagator and construct one possible example for an extension of it. This extension has similar algebraic properties to the single-particle Green's function. We show that it is possible to derive Dyson's equation analogously and define a twoparticle self energy. We examine the properties in relation to the single-particle Green's function. In particular we consider the case of a system interacting with a one-particle potential only. In order to be able to apply the extended propagator to the scattering problem of Section III, we Fourier transform the propagator and Dyson's equation into time space.

\section{A. Single-Particle Green's Function and Dyson Equation}

The single-particle Green's function [1] in energy space is defined by

$$
\begin{aligned}
G_{p q}(\omega)= & \left\langle\Psi_{o}\left|a_{p} \frac{1}{\omega-\hat{H}+E_{o}^{N}+i \eta} a_{q}^{\dagger}\right| \Psi_{o}\right\rangle \\
& +\left\langle\Psi_{o}\left|a_{q}^{\dagger} \frac{1}{\omega-E_{o}^{N}+\hat{H}-i \eta} a_{p}\right| \Psi_{o}\right\rangle .
\end{aligned}
$$

Here, $\left|\Psi_{o}\right\rangle$ denotes the bound ground state of the correlated $N$-fermion system with Energy $E_{o}^{N}$. The operator $a_{q}^{\dagger}$ (or $a_{q}$ ) creates (destroys) one fermion in an orbital that belongs to the one-particle quantum number $q$. The set of possible indices $\{q\}$ covers the one-fermion Hilbert space. The positive infinitesimal $\eta$ provides the correct time ordering when the expressions are Fourier transformed into time space. The Hamiltonian $\hat{H}$ is the usual Fock-space Hamiltonian that contains the kinetic energy as well as possible interactions with external potentials and two-body 
interactions. As usual it is convenient to present it as a sum of a zeroth order part $\hat{H}_{o}$ and an interaction part $\hat{H}_{1}$ :

$$
\hat{H}=\hat{H}_{o}+\hat{H}_{1} .
$$

We choose the zeroth order Hamiltonian to be a diagonal one-particle operator with respect to the underlying orbital basis:

$$
\hat{H}_{o}=\sum_{i} \varepsilon_{i} a_{i}^{\dagger} a_{i}
$$

The two parts of the single-particle Green's function describe the propagation of quasi-particles and quasi-holes in the correlated $N$-fermion system. Formally we can interpret each of the two parts as a projection of an operator resolvent onto the states $a_{p}\left|\Psi_{o}\right\rangle$ and $a_{q}^{\dagger}\left|\Psi_{o}\right\rangle$ respectively. These states belong to the Hilbert spaces of $N \pm 1$ fermions. They are neither orthogonal among each other nor normalized, since the scalar products yield one particle density matrices:

$$
\left\langle\Psi_{o}\left|a_{p} a_{q}^{\dagger}\right| \Psi_{o}\right\rangle \quad \text { or } \quad\left\langle\Psi_{o}\left|a_{q}^{\dagger} a_{p}\right| \Psi_{o}\right\rangle \text {. }
$$

If, however, we formally introduce the composite states

$$
\left|Y_{q}^{G}\right\rangle:=\left(\begin{array}{c}
a_{q}^{\dagger}\left|\Psi_{o}\right\rangle \\
\left\langle\Psi_{o}\right| a_{q}^{\dagger}
\end{array}\right),
$$

these form an orthonormal set with respect to a suitably defined scalar product: ${ }^{1}$

$$
\begin{aligned}
\left\langle Y_{p}^{G} \mid Y_{q}^{G}\right\rangle & =\left\langle\Psi_{o}\left|a_{p} a_{q}^{\dagger}\right| \Psi_{o}\right\rangle+\left\langle\Psi_{o}\left|a_{q}^{\dagger} a_{p}\right| \Psi_{o}\right\rangle \\
& =\left\langle\Psi_{o}\left|\left\{a_{p}, a_{q}^{\dagger}\right\}\right| \Psi_{o}\right\rangle=\delta_{p q} .
\end{aligned}
$$

Mathematically speaking the composite states live in the direct sum of the $(N+1)$ particle Hilbert space and the dual of the $(N-1)$-particle space.

The states $\left|Y_{q}^{G}\right\rangle$ allow the following shorthand notation for the single-particle Green's function:

$$
G_{p q}(\omega)=\left\langle Y_{p}^{G}\left|\frac{1}{\omega-\hat{\mathscr{H}}^{G}}\right| Y_{q}^{G}\right\rangle
$$

${ }^{1}$ We use the following definition for the scalar product, which is canonical:

$$
\left\langle\left(\begin{array}{c}
\left|\psi_{1}\right\rangle \\
\left\langle\psi_{2}\right|
\end{array}\right),\left(\begin{array}{c}
\left|\phi_{1}\right\rangle \\
\left\langle\phi_{2}\right|
\end{array}\right)\right\rangle:=\left\langle\psi_{1} \mid \phi_{1}\right\rangle+\left\langle\phi_{2} \mid \psi_{2}\right\rangle .
$$

The action of a diagonal operator is defined by

$$
\left(\begin{array}{cc}
\hat{A}_{1} & 0 \\
0 & \hat{A}_{2}
\end{array}\right)\left(\begin{array}{c}
\left|\phi_{1}\right\rangle \\
\left\langle\phi_{2}\right|
\end{array}\right):=\left(\begin{array}{c}
\hat{A}_{1}\left|\phi_{1}\right\rangle \\
\left\langle\phi_{2}\right| \hat{A}_{2}
\end{array}\right) .
$$


The operator $\hat{\mathscr{H}}^{G}$ in the composite space is defined by

$$
\hat{\mathscr{H}}^{G}:=\left(\begin{array}{cc}
\hat{H}-E_{o}^{N}-i \eta & 0 \\
0 & E_{o}^{N}-\hat{H}+i \eta
\end{array}\right) .
$$

Eq. (7) now allows us to interpret the full single-particle Green's function as the projection of an operator resolvent onto a set of orthonormal states.

These states $\left\{\left|Y_{q}^{G}\right\rangle\right\}$ span a subspace of the extended Hilbert space they live in. We want to draw attention to the point, that these states are labelled by the complete set of one-particle quantum numbers, regardless, whether or not the indices refer to particle or hole states. Due to this fact, the spanned subspace has the same dimension as (i.e. is isomorphous to) the one-particle Hilbert space. Now we can extend the set $\left\{\left|Y_{q}^{G}\right\rangle\right\}$ to an orthonormal basis $\left\{\left|Q_{J}^{G}\right\rangle\right\}$ of the composite space and use matrix partitioning ${ }^{2}$ in order to derive an expression for the inverse of the matrix $\underline{\underline{G}}(\omega)$ with elements given by Eq. (7). This directly yields Dyson's equation. We present it in the form

$$
\underline{\underline{G}}(\omega)^{-1}=\omega \underline{\underline{1}}-\underline{\underline{\varepsilon}}-\underline{\underline{\Sigma}}(\omega) .
$$

Here the matrix $\underline{\underline{\varepsilon}}$ is diagonal and contains the zeroth order single-particle energies $\varepsilon_{p}$ as well as the infinitsimals $\pm i \eta$. The self energy $\underset{\underline{\Sigma}}{\Sigma}(\omega)$ is defined by

$$
\underline{\underline{\Sigma}}(\omega)=\underline{\mathscr{H}}_{a a}-\underline{\underline{\varepsilon}}+\underline{\mathscr{H}}_{a b} \frac{1}{\omega-\underline{\mathscr{H}}_{b b}} \stackrel{\mathscr{H}}{=}_{b a} .
$$

Here $\stackrel{\mathscr{H}}{=}$ is a matrix representation of the operator $\hat{\mathscr{H}}^{G}$ in the basis $\left\{\left|Q_{J}^{G}\right\rangle\right\} \supset$ $\left\{\left|Y_{q}^{G}\right\rangle\right\}$ of the extended space:

$$
[\stackrel{\mathscr{H}}{=}]_{I J}=\left\langle Q_{I}^{G}\left|\hat{\mathscr{H}}^{G}\right| Q_{J}^{G}\right\rangle .
$$

By virtue of the subdivision of the basis set into two parts, the matrix is structured into blocks:

$$
\stackrel{\mathscr{H}}{=}=\left(\begin{array}{ll}
\mathscr{H}_{a a} & \mathscr{\mathscr { H }}_{a b} \\
\mathscr{\mathscr { H }}_{b a} & \underline{\mathscr{H}}_{b b}
\end{array}\right) .
$$

The block index $a$ refers to the set $\left\{\left|Y_{q}^{G}\right\rangle\right\}$, the index $b$ to the complemental part of the basis. The zeroth order of $\mathscr{\mathscr { H }}_{a a}$ is given by $\underline{\underline{\varepsilon}}$. For more details on the theory we refer to [23].

It depends on the point of view, of course, whether the Dyson equation (9) is seen as an equation that relates quantities which are defined separately or as

\footnotetext{
${ }^{2}$ Instead of the matrix partitioning carried out in [23], one can equally well proceed by introducing projection operators onto the subspace spanned by the set of vectors $\left\{\left|Y_{q}^{G}\right\rangle\right\}$ and its orthogonal complement [12].
} 
a definition of the self energy. In the latter case, it is Eq. (10) that has to be derived. We want to remind the reader, that historically the self energy was defined through its perturbation series and consequently the Dyson equation had to be proven [24, 25]. In contrast to this historic background we understand the Dyson equation rather as an algebraic property of the single-particle Green's function. When we say that we try to construct two-particle propagators that satisfy a Dyson equation, we mean it exactly in this sense.

\section{B. Two-Particle Propagators}

After this excursion to the single-particle Green's function we will focus our attention on two-particle propagators. We consider the particle-particle propagator [15] and its constituents:

$$
\begin{aligned}
& \Pi_{r s, r^{\prime} s^{\prime}}(\omega)=\Pi_{r s, r^{\prime} s^{\prime}}^{+}(\omega)-\Pi_{r s, r^{\prime} s^{\prime}}^{-}(\omega) \\
& \Pi_{r s, r^{\prime} s^{\prime}}^{+}(\omega)=\left\langle\Psi_{o}\left|a_{r} a_{s} \frac{1}{\omega-\hat{H}+E_{o}^{N}+i \eta} a_{s^{\prime}}^{\dagger} a_{r^{\prime}}^{\dagger}\right| \Psi_{o}\right\rangle \\
& \Pi_{r s, r^{\prime} s^{\prime}}^{-}(\omega)=\left\langle\Psi_{o}\left|a_{s^{\prime}}^{\dagger} a_{r^{\prime}}^{\dagger} \frac{1}{\omega-E_{o}^{N}+\hat{H}-i \eta} a_{r} a_{s}\right| \Psi_{o}\right\rangle .
\end{aligned}
$$

The part $\Pi_{r s, r^{\prime} s^{\prime}}^{+}(\omega)$ contains the relevant information for elastic two particle scattering off the correlated ground state, whereas $\Pi_{r s, r^{\prime} s^{\prime}}^{-}(\omega)$ can be used to calculate double ionization spectra. We would like to mention that when transformed to time-space, the particle-particle propagator is just an additive part of the full twoparticle Green's function consisting of two specific time-orders of the latter.

The analogy to the single-particle Green's function fails in the point that neither the $N \pm 2$ particle states

$$
a_{s}^{\dagger} a_{r}^{\dagger}\left|\Psi_{o}\right\rangle \quad \text { or } \quad a_{r} a_{s}\left|\Psi_{o}\right\rangle
$$

nor the composite vectors

$$
\left(\begin{array}{c}
a_{s}^{\dagger} a_{r}^{\dagger}\left|\Psi_{o}\right\rangle \\
\left\langle\Psi_{o}\right| a_{s}^{\dagger} a_{r}^{\dagger}
\end{array}\right)
$$

satisfy any kind of orthogonality relation. Even more, the overlap matrices do not have to be regular at all. This is most easily seen by considering the special case of a noninteracting system (zeroth order). In this case, the states defined above are Slater determinants. All these states, however, vanish, if the index $r$ refers to an orbital that is occupied in the ground state determinant and $s$ refers to an unoccupied orbital, i.e., the space spanned by these states is not isomorphous to the two-particle Hilbert space but has a lower dimension.

Comparing with the one-particle case, we see, that likewise the states $a_{q}^{\dagger}\left|\Psi_{o}\right\rangle$ do not span an isomorphous space to the one-particle space, but the composite states $\left|Y_{q}^{G}\right\rangle$ do. That was the reason for being able to express the single-particle Green's 
function as a matrix inverse or resolvent in a space spanned by the complete set of one-particle quantum numbers. The fact that the states $\left|Y_{q}^{G}\right\rangle$ form an orthonormal set has the consequence that the self energy does not contain any linear contributions in the frequency variable $\omega$.

In order to restore the analogy to the single-particle Green's function we introduce the following extended composite states:

$$
\left|Y_{r s}\right\rangle=\left(\begin{array}{c}
a_{s}^{\dagger} a_{r}^{\dagger}\left|\Psi_{o}\right\rangle \\
\chi_{r s} \\
\left\langle\Psi_{o}\right| a_{s}^{\dagger} a_{r}^{\dagger}
\end{array}\right) .
$$

With the choice

$$
\chi_{r s}:=a_{s}^{\dagger}|\mathrm{vac}\rangle \otimes\left\langle\Psi_{o}\left|a_{r}^{\dagger}-a_{r}^{\dagger}\right| \mathrm{vac}\right\rangle \otimes\left\langle\Psi_{o}\right| a_{s}^{\dagger},
$$

where $|\mathrm{vac}\rangle$ denotes the Fock-space state with no particles, and the "metric"

$$
\hat{\mu}:=\left(\begin{array}{ccc}
1 & 0 & 0 \\
0 & 1 & 0 \\
0 & 0 & -1
\end{array}\right),
$$

these states fulfill the following "orthogonality" relation: ${ }^{3}$

$$
\left\langle Y_{r s}|\hat{\mu}| Y_{r^{\prime} s^{\prime}}\right\rangle=\delta_{r r^{\prime}} \delta_{s s^{\prime}}-\delta_{r s^{\prime}} \delta_{s r^{\prime}} .
$$

This is as much as a unit matrix in a space spanned by two-particle quantum numbers. The second term on the right hand side of Eq. (21) is due to antisymmetry in the one-particle indices that comes from the fermionic nature of the particles.

The extended states live in a composite vector space, denoted by $\mathrm{Y}$, which is the direct sum of the spaces, the three components live in. These are: the $(N+2)$-particle space, the dual of the $(N-2)$-particle space, and the space $\chi_{r s}$ lives in, which is the tensor product of the one particle Hilbert space and the dual of the $(N-1)$-particle space. We have found other possible choices for the extension $\chi_{r s}$ that live in different spaces and utilize different metrics [26]. In this paper we only give one example.

In the composite space $\mathrm{Y}$, the metric $\hat{\mu}$ defines a binary product by

$$
(|A\rangle,|B\rangle)=\langle A|\hat{\mu}| B\rangle .
$$

We want to mention that due to the indefinite choice of the metric $\hat{\mu}$, this binary product is not a scalar product.

${ }^{3}$ For these states the rules of footnote 1 apply likewise. Additionally we define for product states $\left\langle\mid a^{\prime}\right\rangle \otimes\left\langle b^{\prime}|| a,\right\rangle \otimes\langle b \mid\rangle=\left\langle a^{\prime} \mid a\right\rangle \cdot\left\langle b \mid b^{\prime}\right\rangle \quad$ and $\quad(\hat{A} \otimes \hat{B})(|a\rangle \otimes\langle b|)=\hat{A}|a\rangle \otimes\langle b| \hat{B}$. 
In order to define a Green's function analogous to Eq. (7), we first have to define an operator that takes on the role of $\hat{\mathscr{H}}^{G}$ from Eq. (8). A suitable choice for our goals is

$$
\hat{\mathscr{H}}:=\left(\begin{array}{ccc}
\hat{H}-E_{o}^{N}-i \eta & 0 & 0 \\
0 & \hat{H}^{(1)}+E_{o}^{N}-\hat{H}^{(N-1)}+i \eta & 0 \\
0 & 0 & E_{o}^{N}-\hat{H}+i \eta
\end{array}\right) .
$$

The symbol $\hat{H}^{(1)}$ denotes an operator that acts like the usual Fock-space Hamiltonian on the first component (the one-particle part) of the product space, i.e., $\hat{H}^{(1)} \equiv \hat{H} \otimes \hat{1}$. Accordingly, $\hat{H}^{(N-1)}$ acts only on the second component, which is the dual of the $(N-1)$-particle space, i.e., $\hat{H}^{(N-1)} \equiv \hat{1} \otimes \hat{H}$.

Now we are in the position that we can define a new extended two-particle Green's function by

$$
\mathscr{G}_{r s, r^{\prime} s^{\prime}}(\omega):=\left\langle Y_{r s}\left|\hat{\mu} \frac{1}{\omega-\hat{\mathscr{H}}}\right| Y_{r^{\prime} s^{\prime}}\right\rangle .
$$

It follows directly from this definition that this function contains the particleparticle propagator as an additive component:

$$
\begin{aligned}
\mathscr{G}_{r s, r^{\prime} s^{\prime}}(\omega)= & \Pi_{r s, r^{\prime} s^{\prime}}(\omega)+\left\langle\chi_{r s}, \frac{1}{\omega-\hat{H}^{(1)}-E_{o}^{N}+\hat{H}^{(N-1)}-i \eta} \chi_{r^{\prime} s^{\prime}}\right\rangle \\
= & \Pi_{r s, r^{\prime} s^{\prime}}(\omega)+\left\{\frac{1}{i}\left\langle\mathrm{vac}\left|a_{s} \frac{1}{\omega-\hat{H}-i \eta} a_{s^{\prime}}^{\dagger}\right| \mathrm{vac}\right\rangle\right. \\
& \left.*\left\langle\Psi_{o}\left|a_{r^{\prime}}^{\dagger} \frac{1}{\omega-E_{o}^{N}+\hat{H}-i \eta} a_{r}\right| \Psi_{o}\right\rangle\right\} \\
& -\{r \leftrightarrow s\}-\left\{r^{\prime} \leftrightarrow s^{\prime}\right\}+\left\{r \leftrightarrow s, r^{\prime} \leftrightarrow s^{\prime}\right\} .
\end{aligned}
$$

The asterisk $(*)$ denotes convolution with respect to $\omega$ and the symbol $\{r \leftrightarrow s\}$ stands for the term in braces reappearing with interchanged indices.

The new terms that appear in the extended propagator additionally to the particle-particle propagator are analytic functions of $\omega$ in the lower half of the complex plane. Right above the real axis they feature single poles and branch cuts. The poles appear at energies that are sums of single-particle energies (eigenvalues of $\hat{H}^{(1)}$ ) and quasi-hole energies (single-ionization energies or eigenvalues of $\hat{H}^{(N-1)}$ ). Branch cuts originate in the continuous parts of the spectra of the single-particle Hamiltonian $\hat{H}^{(1)}$ and the $(N-1)$-particle Hamiltonian $\hat{H}^{(N-1)}$, respectively. In computations with finite basis sets, of course, only discrete single poles appear. In general, the new poles introduced in the extension $\mathscr{G}(\omega)$ of the particle-particle propagator $\underline{\underline{\Pi}}(\omega)$ are distinct from the poles of the latter. Therefore, the quantities of interest, namely the poles and residues of the particle-particle propagator, can be identified and extracted from the extended propagator. 


\section{Dyson Equation for the Extended Propagator}

The starting point for our considerations is definition (24) and the orthogonality relation (21) for the composite states $\left|Y_{r s}\right\rangle$. Since these states are antisymmetric with respect to a permutation of the indices $r$ and $s$, we introduce the index restriction $r>s$ in order to make the set of vectors $\left\{\left|Y_{r s}\right\rangle\right\}$ linear independent. Now we can extend this set to a basis $\left\{\left|Q_{I}\right\rangle\right\} \supset\left\{\left|Y_{r s}\right\rangle\right\}$ of the space $\mathrm{Y}$. We may choose this set orthogonal with respect to the binary product:

$$
\left\langle Q_{I}|\hat{\mu}| Q_{J}\right\rangle=\delta_{I J} m_{I}
$$

Due to the indefiniteness of the metric $\hat{\mu}$, we cannot normalize all states to $m_{I}=+1$, but we can require that

$$
m_{I} \in\{-1,+1\}
$$

We define the following matrices:

$$
\begin{aligned}
{[\underline{\mu}]_{I J} } & :=\delta_{I J} m_{I}=\left\langle Q_{I}|\hat{\mu}| Q_{J}\right\rangle \\
{[\stackrel{\mathscr{H}}{=}]_{I J}: } & =\left\langle Q_{I}|\hat{\mu} \hat{\mathscr{H}}| Q_{J}\right\rangle .
\end{aligned}
$$

Just like in Section II A, Eq. (12), these matrices are structured into blocks by the subdivision of the basis $\left\{\left|Q_{I}\right\rangle\right\}$ into the subset $\left\{\left|Y_{r s}\right\rangle\right\}$ and its complement. Of course, the upper left block of $\underline{\underline{\mu}}$ is just the unit matrix due to equation (21):

$$
\underline{\underline{\mu}}_{a a}=\underline{\underline{1}}
$$

After some simple manipulations, we can express our extended propagator as the upper left corner of an inverse matrix:

$$
\mathscr{G}_{r s, r^{\prime} s^{\prime}}(\omega)=\left[\frac{1}{\omega \underline{\underline{\mu}}-\underline{\underline{H}}}\right]_{r s, r^{\prime} s^{\prime}} .
$$

By simple matrix partitioning we now find

$$
[\underline{\underline{G}}(\omega)]^{-1}=\omega \underline{\underline{1}}-\underline{\mathscr{H}}_{a a}-\stackrel{\mathscr{H}}{=}_{a b} \frac{1}{\omega_{\underline{\mu}}^{\mu}-\underline{\mathscr{H}}_{b b}} \mathscr{\mathscr { H }}_{b a} .
$$

This equation already reveals the algebraic structure we were aiming at. Of course, we can make it look more like Dyson's equation by introducing the matrix

$$
[\underline{\underline{\varepsilon}}]_{r s, r^{\prime} s^{\prime}}:=\left(\delta_{r r^{\prime}} \delta_{s s^{\prime}}-\delta_{r s^{\prime}} \delta_{s r^{\prime}}\right)\left(\varepsilon_{r}+\varepsilon_{s}\right)
$$


which is easily recognized as the zeroth order of $\mathscr{H}_{a a}$ apart from infinitesimals $\mathcal{O}(\eta)$. When we introduce the "self energy" for the extended propagator by

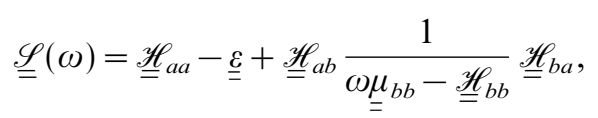

Eq. (33) becomes Dyson's equation for the extended two-particle propagator:

$$
[\underline{\underline{G}}(\omega)]^{-1}=\omega \underline{\underline{1}}-\underline{\underline{\varepsilon}}-\underline{\underline{\mathscr{P}}}(\omega) .
$$

Obviously, the zeroth order of the extended propagator takes on the following appearance:

$$
\left[\underline{\underline{G}}^{(0)}(\omega)\right]^{-1}=\omega \underline{\underline{1}}-\underline{\underline{\varepsilon}},
$$

where the infinitesimal $\operatorname{in}\left(2 \bar{n}_{r} \bar{n}_{s}-1\right)$ has been left out for simplicity. The antioccupation number $\bar{n}_{r}$ takes on the value 1 if the index $r$ refers to an orbital unoccupied in the zeroth order ground state (virtual orbital) and 0 if otherwise.

Finally we can express the Dyson equation (36) in the appealing equivalent form:

$$
\underline{\underline{\mathscr{G}}}(\omega)=\underline{\underline{\mathscr{G}}}^{(0)}(\omega)+\underline{\underline{\mathscr{G}}}^{(0)}(\omega) \underline{\underline{\mathscr{P}}}(\omega) \underline{\underline{\mathscr{G}}}(\omega) .
$$

We note that the self energy $\mathscr{\mathscr { S }}(\omega)$ can be calculated from Eq. (35). Of course, the choice of the extended basis is not unique. Different ways of constructing finite subsets of this basis will hence lead to different approximations for the self energy. For example, powerful approximations may be achieved by constructing the basis set from correlated excited states, classified by the nature of the excitation with respect to the ground state. This is a common technique used in many modern many-body methods [27, 28].

By construction, the extended Green's function $\underline{\underline{G}}(\omega)$ contains the full particleparticle propagator and, therefore, it can be used not only for scattering but also for calculating double-ionization energies and the corresponding transition amplitudes. One has to be careful, however, when employing perturbation theory to extract approximations to the self energy $\mathscr{\mathscr { S }}(\omega)$ for this purpose. The reason is that there are states in the extended basis contributing to $\mathscr{\mathscr { H }}_{b b}$ that are degenerate in zeroth order with states $\left|Y_{r s}\right\rangle$ where $r$ and $s$ are hole indices (i.e., assign occupied orbitals in the unperturbed ground state). It is, of course, possible to obtain valid approximations by applying the formalism of degenerate perturbation analysis. The deeper reason for the appearance of these degeneracies lies in the indefinite choice of the metric $\hat{\mu}$ in Eq. (20). It is an open question, whether suitable extensions $\chi_{r s}$ in (18) can be found that avoid the degeneracies by using a definite metric. For a more detailed discussion of the properties of $\underline{\underline{G}}(\omega)$, of perturbation theory, and of different choices of extensions $\chi_{r s}$ see reference [26]. 


\section{Resolvents and Energy Dependence}

A striking new property of the extended propagator is revealed when we compare the various one- and two-particle propagators and try to interpret them as resolvents of some operator. We remark that, of course, all the matrices in the twoparticle quantum numbers $(r, s)$ are not matrices in a literal sense, since they are in general of infinite dimension and the indices may be continuous. These matrices can rather be understood as linear operators in the two particle Hilbert space. The matrix $\underline{\varepsilon}$ is then a representation of the zeroth order Hamiltonian $\hat{H}_{o}$, restricted to the two particle space. In this sense, the zeroth order propagator $\underline{\underline{G}}^{(0)}(\omega)$ is a resolvent of this Hamiltonian, as Eq. (37) shows. Of course, the full extended propagator $\underline{\underline{G}}(\omega)$ can be seen as a resolvent of the energy-dependent operator

$$
\underline{\underline{\varepsilon}}+\underline{\underline{\mathscr{P}}}(\omega) \text {. }
$$

In Section III E we will see that indeed this operator is an effective two-particle Hamiltonian that can be used to describe elastic two-particle scattering off a correlated $N$-fermion target. We want to remind the reader that neither the particleparticle propagator $\underline{\underline{\Pi}}(\omega)$, nor one of its components $\underline{\underline{\Pi}}^{ \pm}(\omega)$ is a resolvent in the complete two-particle space, like the discussion at the beginning of II B shows. Still, in a restricted space these propagators may be understood as resolvents. But the energy-dependent effective Hamiltonians that could be derived this way are pathological, since they contain linear contributions in $\omega$ that diverge at high energies. The energy dependence of the two-particle self energy $\underline{\underline{\mathscr{S}}}(\omega)$, on the other hand, is given by Eq. (35), where the analytic structure is determined through a partial fraction series. For practical purposes this implies that the self energy has a finite limit for values of the energy variable $\omega$ far outside the spectrum of the Hamiltonian.

\section{E. One-Particle Potentials}

Another new feature of the extended two-particle propagators concerns the behaviour with respect to one-particle potentials. For a system interacting with one-particle potentials the Hamiltonian $\hat{H}$ is a one-particle operator, i.e. it can be written in the form

$$
\hat{H}=\sum_{i j} h_{i j} a_{i}^{\dagger} a_{j} .
$$

In this case, the particles of the system do not interact by real two-body forces but only with external or mean field potentials. Any operator of the form (39) can be diagonalized (i.e. $h_{i j} \propto h_{i} \delta_{i j}$ ) by a simple unitary transformation $t_{i j}$ of the set of orbitals (basis of one-particle wavefunctions). In general, this transformation includes the full one-particle space. It can be seen directly from the definitions (24), (1), (13), (14), and (15), that all of the propagators we have introduced so far, transform 
with one unitary transformation matrix on each one-particle index. For example, the particle-particle propagator transforms like

$$
\Pi_{i j, k l}^{\prime}=\sum_{i^{\prime} j^{\prime} k^{\prime} l^{\prime}} t_{i i^{\prime}}^{*} t_{j j^{\prime}}^{*} \Pi_{i^{\prime} j^{\prime}, k^{\prime} l^{\prime}} t_{k^{\prime} k} t_{l^{\prime} l}
$$

Since a diagonalized one-particle Hamiltonian is equivalent to the zeroth order Hamiltonian (i.e. it can be written in the form of Eq. (3)), we can easily study the behaviour of the various propagators under the influence of a one-particle potential by performing the inverse transformation on the zeroth order propagators. It is particularly interesting to observe what happens under this procedure to equations (37) and (36) for our extended two-particle propagator, and Eq. (9) for the singleparticle Green's function. From the argumentation given above, it follows immediately that in both cases the self energies $\underline{\underline{\mathscr{S}}}(\omega)$ and $\underline{\underline{\Sigma}}(\omega)$ become energyindependent for one-particle potentials. It is easy to see that they are exact already in first order in the one-body interaction. For completeness we give $\underline{\underline{\Sigma}}(\omega)$ and $\underline{\underline{\mathscr{P}}}(\omega)$ explicitly in these cases:

$$
\begin{aligned}
\Sigma_{p q}(\omega) & =h_{p q} \\
\mathscr{S}_{r s, r^{\prime} s^{\prime}}(\omega) & =h_{r r^{\prime}} \delta_{s s^{\prime}}+h_{s s^{\prime}} \delta_{r r^{\prime}}-h_{r s^{\prime}} \delta_{s r^{\prime}}-h_{s r^{\prime}} \delta_{r s^{\prime}} .
\end{aligned}
$$

These matrices can be seen as representations of the one-particle Hamiltonian in the spaces spanned by one-particle wave functions $a_{q}^{\dagger}|\mathrm{vac}\rangle$ and two-particle Slater determinants $a_{r^{\prime}}^{\dagger} a_{s^{\prime}}^{\dagger}|\mathrm{vac}\rangle$, respectively.

Speaking in terms of resolvents, we see that both our extended two-particle propagator as well as the single-particle Green's function now become resolvents of energy independent effective Hamiltonians and these Hamiltonians contain no higher than first order contributions in the interaction. We want to stress that this is not the case for the particle-particle propagator $\underline{\underline{\Pi}}(\omega)$ from (13) or any of its constituents $\underline{\underline{\Pi}}^{ \pm}(\omega)$. We already pointed out that these propagators can be viewed as resolvents only in restricted index spaces. Therefore, the behaviour of their matrix inverses under transformations of the orbital basis is complicated and leads to energy-dependent effective Hamiltonians with contributions in all orders of perturbation theory. For this reason the calculation of scattering amplitudes or doubleionization energies via the particle-particle propagator or its constituents in the presence of one-particle potentials is formally nearly as complicated as the treatment of a fully correlated system (as long as one does not transform to new orbitals, of course). When using our new extended two-particle propagators, however, already the first order contribution to its self energy yields the influence of the oneparticle potentials exactly.

\section{F. Time Space Equations}

For the sake of conceptual clearness we have developed the algebra of the extended two-particle propagators in energy space. In order to apply our developments to 
the scattering problem of Section III, we will consider briefly the Fourier transforms into time space. We use the following convention for Fourier transformation:

$$
f(t):=\int_{-\infty}^{\infty} \frac{d \omega}{2 \pi} e^{-i \omega t} f(\omega)
$$

In order to agree with standard notation we define

$$
\begin{gathered}
\mathscr{G}_{r s, r^{\prime} s^{\prime}}\left(t, t^{\prime}\right):=\mathscr{G}_{r s, r^{\prime} s^{\prime}}\left(t-t^{\prime}\right) \\
\Pi_{r s, r^{\prime} s^{\prime}}\left(t, t^{\prime}\right):=\Pi_{r s, r^{\prime} s^{\prime}}\left(t-t^{\prime}\right) .
\end{gathered}
$$

When transforming the extended two particle propagator $\mathscr{C}_{r s, r^{\prime} s^{\prime}}(\omega)$, it is most convenient to start from Eq. (26) and make use of the convolution theorem. The result reads

$$
\begin{aligned}
\mathscr{G}_{r s, r^{\prime} s^{\prime}}\left(t, t^{\prime}\right)= & \Pi_{r s, r^{\prime} s^{\prime}}\left(t, t^{\prime}\right)+\theta\left(t^{\prime}-t\right)\left\{\frac{1}{i}\left\langle\operatorname{vac}\left|a_{s}(t) a_{s^{\prime}}^{\dagger}\left(t^{\prime}\right)\right| \mathrm{vac}\right\rangle\right. \\
& \left.\times\left\langle\Psi_{o}\left|a_{r^{\prime}}^{\dagger}\left(t^{\prime}\right) a_{r}(t)\right| \Psi_{o}\right\rangle\right\}-\{r \leftrightarrow s\}-\left\{r^{\prime} \leftrightarrow s^{\prime}\right\}+\left\{r \leftrightarrow s, r^{\prime} \leftrightarrow s^{\prime}\right\},
\end{aligned}
$$

where the Fourier transform of the particle-particle propagator from Eq. (13) is given by

$$
\begin{aligned}
\Pi_{r s, r^{\prime} s^{\prime}}\left(t, t^{\prime}\right)= & \frac{1}{i} \theta\left(t-t^{\prime}\right)\left\langle\Psi_{o}\left|a_{r}(t) a_{s}(t) a_{s^{\prime}}^{\dagger}\left(t^{\prime}\right) a_{r^{\prime}}^{\dagger}\left(t^{\prime}\right)\right| \Psi_{o}\right\rangle \\
& -\frac{1}{i} \theta\left(t^{\prime}-t\right)\left\langle\Psi_{o}\left|a_{s^{\prime}}^{\dagger}\left(t^{\prime}\right) a_{r^{\prime}}^{\dagger}\left(t^{\prime}\right) a_{r}(t) a_{s}(t)\right| \Psi_{o}\right\rangle .
\end{aligned}
$$

The two terms on the right hand side of the above equation represent the parts $\Pi_{r s, r^{\prime} s^{\prime}}^{+}\left(t, t^{\prime}\right)$ and $\Pi_{r s, r^{\prime} s^{\prime}}^{-}\left(t, t^{\prime}\right)$, respectively. In these expressions the operators appear in the Heisenberg picture with a time dependence given by

$$
\hat{A}(t)=e^{i \hat{H} t} \hat{A} e^{-i \hat{H} t} .
$$

Heavyside's theta function is defined as follows:

$$
\theta(t):=\left\{\begin{array}{ll}
1 & \text { for } t \geqslant 0 \\
0 & \text { for } t<0
\end{array} .\right.
$$

As usual, the signs of the infinitesimals \pm i bring about the time ordering via theta functions. Although the time ordering does have relevance in scattering by giving 
the right boundary conditions, it does not affect the algebraic properties, since Dyson's equation transforms, regardless of the chosen time ordering of $\underline{\underline{G}}$, into

$$
\begin{aligned}
\mathscr{G}_{r s, r^{\prime} s^{\prime}}\left(t, t^{\prime}\right)= & \mathscr{G}_{r s, r^{\prime} s^{\prime}}^{(0)}\left(t, t^{\prime}\right)+\sum_{\substack{p q \\
p^{\prime} q^{\prime}}} \int_{-\infty}^{\infty} d t_{1} \int_{-\infty}^{\infty} d t_{2} \mathscr{G}_{r s, p q}^{(0)}\left(t, t_{1}\right) \\
& \times \mathscr{S}_{p q, p^{\prime} q^{\prime}}\left(t_{1}, t_{2}\right) \mathscr{G}_{p^{\prime} q^{\prime}, r^{\prime} s^{\prime}}\left(t_{2}, t^{\prime}\right) .
\end{aligned}
$$

The zeroth order propagator in time space reads

$$
\mathscr{G}_{r s, r^{\prime} s^{\prime}}^{(0)}\left(t, t^{\prime}\right)=\left(\delta_{r r^{\prime}} \delta_{s s^{\prime}}-\delta_{r s^{\prime}} \delta_{s r^{\prime}}\right) \frac{1}{i} e^{i\left(\varepsilon_{r}+\varepsilon_{s}\right)\left(t-t^{\prime}\right)}\left[\bar{n}_{r} \bar{n}_{s} \theta\left(t-t^{\prime}\right)-\left(1-\bar{n}_{r} \bar{n}_{s}\right) \theta\left(t^{\prime}-t\right)\right] .
$$

As long as $\eta$ in (23) is non-zero, the Fourier transform of the self energy defined through (35) and (30) is unambiguous. Like with the Green's function, different signs in (23) lead to a different self energy in time space although the Dyson equation (48) remains valid.

\section{OPTICAL MODEL DESCRIPTION OF TWO-PARTICLE SCATTERING}

In this chapter we will show how an extended two-particle propagator that satisfies Dyson's equation can be used to derive effective two-particle equations for the elastic scattering of a two-fermion projectile by a correlated many-body target. ${ }^{4}$ The extended propagator introduced in the preceding chapter serves as one example, but other two-particle Green's functions can be used as well, provided they fulfill certain requirements which will be discussed. We will show how the improper self energy of this propagator can be identified with the scattering $T$-matrix. Furthermore, we will derive an effective Lippmann-Schwinger equation and an eigenvalue equation involving a two-particle scattering amplitude and an optical potential that is given by the two-particle self energy.

\section{A. The Scattering Problem}

The type of scattering process that can be straightforwardly described by the considered type of two-particle propagators is the elastic scattering of a two-particle projectile off a correlated many-body target. For simplicity we assume that all particles are indistinguishable fermions, although extensions to composite particle systems, e.g. a positronium projectile, are easy to do and usually simplify the actual calculations. The target is taken to be in its nondegenerate $N$-fermion ground state

\footnotetext{
${ }^{4}$ For earlier work on effective two-particle scattering equations in a different context see [21].
} 
$\left|\Psi_{o}\right\rangle$ with energy $E_{o}^{N}$. In the simplest case, the projectile may consist of two (asymptotically) free fermions and is denoted by

$$
a_{p}^{\dagger} a_{q}^{\dagger}|\mathrm{vac}\rangle \text {. }
$$

It is an eigenstate of the "free particle" Hamiltonian which can be identified with the zeroth order Hamiltonian $\hat{H}_{o}$ from Eq. (3). All relevant information on the scattering process is now contained in the two particle part $\underline{\underline{\Pi}}^{+}(\omega)$ of the particleparticle propagator from Eq. (14). However, we may consider a more general projectile consisting of an arbitrary two particle eigenstate $|K\rangle$ of some "free projectile" Hamiltonian $\hat{H}_{p}$.

$$
\begin{aligned}
\hat{H}_{p}|K\rangle & =\mathscr{E}_{K}|K\rangle \\
|K\rangle & :=A_{K}^{\dagger}|\mathrm{vac}\rangle
\end{aligned}
$$

with $A_{K}^{\dagger}$ denoting the projectile creation operator:

$$
A_{K}^{\dagger}=\int d p d q \phi_{K}(p, q) a_{p}^{\dagger} a_{q}^{\dagger}
$$

Here the cumulative index $K$ denotes a complete set of quantum numbers that characterize the two-particle state $|K\rangle$ with energy $\mathscr{E}_{K}$. Let $p$ and $q$ denote one-particle quantum numbers, e.g. momentum and spin $p=(\mathbf{p}, \sigma)$. Since it appears more natural to use continuous sets of quantum numbers rather than discrete orbital indices when describing scattering processes, we changed the notation and replaced sums over one- or two-particle indices by integrals. This is merely a formal step, of course, since the integrals still contain discrete sums, e.g. over spin indices. The operators $a_{p}^{\dagger}$ and $a_{q}^{\dagger}$ create one-particle states with the given quantum numbers. The quantity $\phi_{K}(p, q)$ plays the role of a two-particle wavefunction.

The simple case of a projectile consisting of two free particles now emerges as a special case in the more general treatment. The free projectile Hamiltonian $\hat{H}_{p}$ reduces to the kinetic energy operator and we can take $a_{p}^{\dagger}$ and $a_{q}^{\dagger}$ to create plane wave states. In that case we can choose the wavefunction to be of the form

$$
\phi_{K}(p, q)=\delta_{k_{1} p} \delta_{k_{2} q},
$$

where $K \equiv\left(k_{1}, k_{2}\right)$. In the following we will develop the theory for a general projectile state, but come back to the simpler case at times.

The principal quantity for describing the results of scattering experiments is the so called scattering matrix or S-matrix:

$$
S\left(K \leftarrow K^{\prime}\right)=\left\langle\Psi_{K}^{-} \mid \Psi_{K^{\prime}}^{+}\right\rangle .
$$

We want to describe the elastic scattering of two-particle states $|K\rangle$. The $S$-matrix then gives the probability amplitude for the scattering of the two-particle projectile 
$\left|K^{\prime}\right\rangle$ off the target state $\left|\Psi_{o}\right\rangle$, into the projectile state $|K\rangle$, the target remaining in the state $\left|\Psi_{o}\right\rangle$. Formula (54) gives the $S$-matrix as the overlap of the so called scattering states:

$$
\left|\Psi_{K}^{ \pm}\right\rangle=\lim _{t \rightarrow \mp \infty} A_{K}^{\dagger}(t)\left|\Psi_{o}\right\rangle e^{-i \mathscr{E}_{K} t}
$$

Here the projectile creation operator $A_{K}^{\dagger}$ appears in the Heisenberg picture defined in Eq. (46). The scattering states can be understood as states in the Heisenberg picture that behave asymptotically in the distant past/future like a free projectile $|K\rangle$ and target $\left|\Psi_{o}\right\rangle$.

Strictly speaking, the limit in (55) does not converge in the usual sense and is meant as an Abelian limit [29]:

$$
\left|\Psi_{K}^{ \pm}\right\rangle=\lim _{\varepsilon \searrow 0} \int_{0}^{\mp \infty} d \tau e^{ \pm \varepsilon \tau} A_{K}^{\dagger}(\tau)\left|\Psi_{o}\right\rangle e^{-i \mathscr{E}_{K} \tau}
$$

In the following we will use the Abelian limit where appropriate, without referring explicitly to it.

For the $S$-matrix we find

$$
S\left(K \leftarrow K^{\prime}\right)=\lim _{\substack{t \rightarrow \infty \\ t^{\prime} \rightarrow-\infty}} e^{i \mathscr{E}_{K} t}\left\langle\Psi_{o}\left|A_{K}(t) A_{K^{\prime}}^{\dagger}\left(t^{\prime}\right)\right| \Psi_{o}\right\rangle e^{-i \mathscr{E}_{K^{\prime}} t^{\prime}} .
$$

Employing the definition (52) of the projectile creation operator $A_{K}^{\dagger}$, we arrive at the following expression:

$$
\begin{aligned}
S\left(K \leftarrow K^{\prime}\right)= & \lim _{\substack{t \rightarrow \infty \\
t^{\prime} \rightarrow-\infty}} e^{i\left(\mathscr{E}_{K} t-\mathscr{E}_{K^{\prime}} t^{\prime}\right)} \int d p d q d p^{\prime} d q^{\prime} \phi_{K}^{*}(p, q) \\
& \times\left\langle\Psi_{o}\left|a_{q}(t) a_{p}(t) a_{p^{\prime}}^{\dagger}\left(t^{\prime}\right) a_{q^{\prime}}^{\dagger}\left(t^{\prime}\right)\right| \Psi_{o}\right\rangle \phi_{K^{\prime}}\left(p^{\prime}, q^{\prime}\right) \\
= & \lim _{\substack{t \rightarrow \infty \\
t^{\prime} \rightarrow-\infty}} e^{i\left(\mathscr{E}_{K} t-\mathscr{E}_{K^{\prime}} t^{\prime}\right)} \int d p d q d p^{\prime} d q^{\prime} \cdot \phi_{K}^{*}(p, q) \Pi_{p q, p^{\prime} q^{\prime}}^{+}\left(t, t^{\prime}\right) \phi_{K^{\prime}}\left(p^{\prime}, q^{\prime}\right)
\end{aligned}
$$

The last equality follows from definition (45). This shows that the two-particle part $\underline{\Pi}^{+}\left(t, t^{\prime}\right)$ of the particle-particle propagator contains all relevant information on the scattering process. Obviously, $\underline{\underline{\Pi}}^{+}\left(t, t^{\prime}\right)$ can be replaced by $\underline{\underline{G}}\left(t, t^{\prime}\right)$ as well as by $\underline{\underline{\Pi}}\left(t, t^{\prime}\right)$, since the additional terms do not contribute due to the fixed time ordering in equation (58), like it can be seen from equations (44) and (45).

\section{B. General Requirements for a Scattering Propagator}

The main objective of this chapter is to show how the extended two-particle propagator $\underline{\underline{G}}$ of Eq. (24) or (44) can be used to reduce the many-body scattering problem to effective two-particle scattering equations. The derivation we will give 
in the following, however, only makes use of some fundamental properties of the extended propagator. Loosely speaking these are: the propagator used contains $\underline{\underline{\Pi}}^{+}$ and satisfies Dyson's equation. Since it is possible to find different extensions of the particle-particle propagator that meet these requirements, we will introduce the new symbol $\widetilde{\mathscr{G}}_{K, K^{\prime}}\left(t, t^{\prime}\right)$ for a general propagator that fulfills certain requirements stated below. We will refer to the general two-particle Green's function $\widetilde{\mathscr{G}}_{K, K^{\prime}}\left(t, t^{\prime}\right)$ as the scattering propagator. For convenience this propagator is labelled with two-particle quantum numbers like $K$ and $K^{\prime}$, each of these replacing two orbital indices. The transformation between these two methods of labelling two particle propagators is given by the two particle wavefunctions $\phi_{K}(p, q)$ like in Eq. (52). In the following we will state the required properties explicitly.

The first requirement allows one to rewrite Eq. (57) for the $S$-matrix in terms of the scattering propagator:

(i) For $t>t^{\prime}$ the scattering propagator reduces to the transformed twoparticle propagator $\underline{\underline{\Pi}}^{+}\left(t, t^{\prime}\right)$ :

$$
i \tilde{\mathscr{G}}_{K, K^{\prime}}\left(t, t^{\prime}\right)=\left\langle\Psi_{o}\left|A_{K}(t) A_{K^{\prime}}^{\dagger}\left(t^{\prime}\right)\right| \Psi_{o}\right\rangle \quad \text { for } t>t^{\prime} .
$$

The second property requires the main new feature of our extended two-particle propagator, Dyson's equation. It introduces the self energy $\widetilde{\mathscr{S}}$ of the scattering propagator $\tilde{\mathscr{G}}$, which will serve as the two-particle optical potential.

(ii) The scattering propagator satisfies a Dyson equation:

$$
\begin{aligned}
\tilde{\mathscr{G}}_{K, K^{\prime}}\left(t, t^{\prime}\right)= & \widetilde{\mathscr{G}}_{K, K^{\prime}}^{(p)}\left(t, t^{\prime}\right)+\int d K_{1} d K_{2} d t_{1} d t_{2} \widetilde{\mathscr{G}}_{K, K_{1}}^{(p)}\left(t, t_{1}\right) \\
& \times \tilde{\mathscr{T}}_{K_{1}, K_{2}}\left(t_{1}, t_{2}\right) \tilde{\mathscr{G}}_{K_{2}, K^{\prime}}\left(t_{2}, t^{\prime}\right) .
\end{aligned}
$$

Here, a free projectile propagator $\tilde{\mathscr{G}}^{(p)}$ appears that reads

$$
i \tilde{\mathscr{G}}_{K, K^{\prime}}^{(p)}\left(t, t^{\prime}\right)=\theta\left(t-t^{\prime}\right) \delta_{K, K^{\prime}} e^{i \mathscr{E}_{K}\left(t-t^{\prime}\right)}
$$

for $K$ or $K^{\prime}$ belonging to the subset of two-particle quantum numbers used to describe the projectile states.

The integrals over $K_{i}$ in (61) are meant to run over the complete two particle space. The $\delta$ function denotes a proper normalization condition. The actual form of this condition depends on the set of quantum numbers used. In the example of two free projectile particles this normalization condition reads

$$
\delta_{K, K^{\prime}}=\delta_{k_{1} k_{1}^{\prime}} \delta_{k_{2} k_{2}^{\prime}}-\delta_{k_{1} k_{2}^{\prime}} \delta_{k_{2} k_{1}^{\prime}} .
$$

The second term on the right hand side accounts for the fermionic nature of the projectile's components. In the case of a bound state projectile, on the other hand, 
the index $K$ may combine an internal quantum number $n$ of the projectile with a center of mass wavevector $\mathbf{k}$ and consequently the $\delta$ function is given by

$$
\delta_{K, K^{\prime}}=\delta_{n n^{\prime}} \delta_{\mathbf{k k}^{\prime}}
$$

The third requirement is a minor technical point. It is a common feature of many-body Green's functions depending on two time variables that these functions actually depend only on the time difference, when the total energy of the given system is conserved. Thus we require:

(iii) As a consequence of the conservation of energy we want the function $\tilde{\mathscr{G}}$ and the other functions that appear in the Dyson equation to depend only on the time difference $t-t^{\prime}$.

It is easy to see that these conditions are fulfilled by the extended propagator $\mathscr{G}_{k_{1} k_{2}, k_{1}^{\prime} k_{2}^{\prime}}\left(t, t^{\prime}\right)$ of Eq. (44), in the case of a projectile consisting of two (asymptotically) free particles where $K \equiv\left(k_{1}, k_{2}\right)$ holds. The zeroth order propagator $\mathscr{G}_{k_{1} k_{2}, k_{1}^{\prime} k_{2}^{\prime}}^{(0)}\left(t, t^{\prime}\right)$ from Eq. (49) now can be identified with the free projectile propagator $\tilde{\mathscr{G}}_{K, K^{\prime}}^{(p)}\left(t, t^{\prime}\right)$.

In the more general case of a projectile in an arbitrary, possibly bound, twoparticle state, the transformed function

$$
\tilde{\mathscr{G}}_{K, K^{\prime}}\left(t, t^{\prime}\right):=\int d p d q d p^{\prime} d q^{\prime} \phi_{K}^{*}(p, q) \mathscr{G}_{p q, p^{\prime} q^{\prime}}\left(t, t^{\prime}\right) \phi_{K^{\prime}}\left(p^{\prime}, q^{\prime}\right)
$$

fulfills the requirements. We only have to be a little more careful, if the projectile state $|K\rangle$ is not a Slater determinant and thus cannot be expressed as the eigenstate of a one-particle operator. This is generally true for bound two-particle projectile states. Since the zeroth order Hamiltonian $\hat{H}_{o}$ of Eq. (3) is usually required to be a one-particle operator, the free projectile propagator $\tilde{\mathscr{G}}^{(p)}$ of Eq. (62) will generally differ from the zeroth order $\mathscr{G}^{(0)}$ of Eq. (49) both in the phases and the set of quantum numbers that diagonalizes the propagator. This does not cause a serious problem, however, since the Dyson equation (48) for $\mathscr{G}$ can be rewritten with $\mathscr{G}^{(0)}$ replaced by the propagator $\widetilde{\mathscr{G}}^{(p)}$. This implies a trivial renormalization of the $\omega$ independent part of the self energy $\underline{\underline{\mathscr{S}}}(\omega)$, as the energy-space equations $(36)$ and (38) show.

If we still want to keep the direct connection to perturbation theory, such that $\tilde{\mathscr{G}}^{(p)}$ is the zeroth order of $\widetilde{\mathscr{G}}$, we have to find some way to include $\hat{H}_{p}$, which is primarily an operator in the two-particle Hilbert space, into the zeroth order (Fock-space) Hamiltonian $\hat{H}_{o}$. In this case we have to take care that the free projectile states do not interfere with the zeroth order ground state of the target, which also has to be an eigenstate of $\hat{H}_{o}$. This could be done by constructing the projectile from unoccupied (virtual) orbitals only. A procedure similar to this is described in reference [21]. 
We now carry on without referring to the extended propagator introduced in the last chapter but assume that we have a Green's function, the scattering propagator $\widetilde{\mathscr{G}}$, which possesses the properties stated above.

\section{Relation to the Scattering T-Matrix}

Now we are in the position to link up with Eq. (57) and express the $S$-matrix in terms of the scattering propagator using property (i):

$$
S\left(K \leftarrow K^{\prime}\right)=\lim _{\substack{t \rightarrow \infty \\ t^{\prime} \rightarrow-\infty}} i \widetilde{\mathscr{G}}_{K, K^{\prime}}\left(t, t^{\prime}\right) e^{i\left(\mathscr{E}_{K} t-\mathscr{E}_{K} t^{\prime}\right)} .
$$

In order to see the connection to the popular $T$-matrix description of scattering we rewrite Dyson's equation (61) introducing the improper self energy or Green's function $T$-matrix $\tilde{\mathscr{T}}_{K, K^{\prime}}\left(t, t^{\prime}\right)$ :

$$
\begin{aligned}
& \underline{\underline{\mathscr{G}}}=\underline{\underline{\mathscr{G}}}^{(p)}+\underline{\underline{\mathscr{G}}}^{(p)} \underline{\mathscr{T}}^{\widetilde{\mathscr{G}}}{ }^{(p)} \\
& \underline{\underline{\mathscr{T}}}=\underline{\underline{\mathscr{T}}}+\underline{\underline{\mathscr{T}}} \underline{\underline{\mathscr{G}}}^{(p)} \stackrel{\widetilde{\mathscr{T}}}{=} .
\end{aligned}
$$

Here, we are using a shorthand notation where the entities like $\underline{\underline{\mathscr{G}}}$ are matrices with indices $(K, t)$ and $\left(K^{\prime}, t^{\prime}\right)$. The matrix product then implies integration over all inner indices, namely the two particle quantum numbers and the time variable. For example,

$$
\begin{aligned}
{\left[\underline{\underline{\mathscr{G}}}^{(p)} \underline{\underline{\mathscr{T}}} \underline{\underline{\mathscr{\mathscr { G }}}}(p)\right]_{(K, t)\left(K^{\prime}, t^{\prime}\right)}=} & \int d K_{1} d K_{2} d t_{1} d t_{2} \tilde{\mathscr{G}}_{K, K_{1}}^{(p)}\left(t, t_{1}\right) \\
& \times \tilde{\mathscr{T}}_{K_{1}, K_{2}}\left(t_{1}, t_{2}\right) \tilde{\mathscr{G}}_{K_{2}, K^{\prime}}^{(p)}\left(t_{2}, t^{\prime}\right) .
\end{aligned}
$$

It is easily found that inserting (67) into the expression (66) for the $S$-matrix and using property (i) yields

$$
S\left(K \leftarrow K^{\prime}\right)=\delta_{K K^{\prime}}-2 \pi i \tilde{T}_{K, K^{\prime}}\left(\mathscr{E}_{K}\right) \delta\left(\mathscr{E}_{K}-\mathscr{E}_{K^{\prime}}\right) .
$$

Here, the quantity $\tilde{\mathscr{T}}_{K, K^{\prime}}\left(\mathscr{E}_{K}\right)$ denotes the Fourier transform of the improper self energy $\tilde{\mathscr{T}}_{K, K^{\prime}}\left(t, t^{\prime}\right)$, evaluated at energy $\mathscr{E}_{K}$. Transforming $\tilde{\mathscr{T}}$ on a single energy variable becomes possible, because the improper self energy is a function of the time difference only. This is a consequence of property (iii).

Eq. (69) now allows us to identify the improper self energy $\tilde{\mathscr{T}}_{K, K^{\prime}}\left(\mathscr{E}_{K}\right)$ with the on-shell scattering $T$-matrix $t\left(K \leftarrow K^{\prime}\right)$, which is defined by

$$
S\left(K \leftarrow K^{\prime}\right)=\delta_{K K^{\prime}}-2 \pi i \delta\left(\mathscr{E}_{K}-\mathscr{E}_{K^{\prime}}\right) t\left(K \leftarrow K^{\prime}\right) .
$$

This shows a connection between the many-body Green's function $\tilde{\mathscr{G}}$ and effective scattering quantities represented by the common scattering $S$ - and $T$-matrices, 
which is analogous to the problem of scattering one fermion off a correlated many fermion target $[30,31]$. In that case the single-particle Green's function $\underline{\underline{G}}\left(t, t^{\prime}\right)$ takes on the role of $\underline{\underline{\mathscr{G}}}\left(t, t^{\prime}\right)$.

\section{Effective Lippmann-Schwinger Equation}

We will now proceed to further carry this analogy and derive effective twoparticle scattering equations. First we define scattering amplitudes that take the role of effective two-particle wavefunctions:

$$
f^{K^{\prime}+}(K, t):=\left\langle\Psi_{o}\left|A_{K}(t)\right| \Psi_{K^{\prime}}^{+}\right\rangle .
$$

These amplitudes contain all the relevant information concerning the scattering process, since we can express the $S$-matrix through

$$
S\left(K \leftarrow K^{\prime}\right)=\lim _{t \rightarrow \infty} e^{i \mathscr{E}_{K} t} f^{K^{\prime}+}(K, t) .
$$

The relation to the Green's function is given by

$$
f^{K^{\prime}+}(K, t)=\lim _{t^{\prime} \rightarrow-\infty} i \widetilde{\mathscr{G}}_{K, K^{\prime}}\left(t, t^{\prime}\right) e^{-i \mathscr{E}_{K^{\prime}} t^{\prime}}
$$

The time dependence of the scattering amplitudes can be taken from the definition, using the fact that $\left|\Psi_{o}\right\rangle$ and $\left|\Psi_{K^{\prime}}^{+}\right\rangle$are eigenfunctions of the Hamiltonian:

$$
\begin{aligned}
f^{K^{\prime}+}(K, t) & =\left\langle\Psi_{o}\left|A_{K}(t)\right| \Psi_{K^{\prime}}^{+}\right\rangle \\
& =\left\langle\Psi_{o}\left|e^{i \hat{H} t} A_{K} e^{-i \hat{H} t}\right| \Psi_{K^{\prime}}^{+}\right\rangle \\
& =e^{-i \mathscr{E}_{K^{\prime}} t} f^{K^{\prime}+}(K) .
\end{aligned}
$$

The last equality defines the time-independent scattering amplitude. We note that

$$
\begin{aligned}
f^{K^{\prime}+}(K) & =e^{i \mathscr{E}_{K^{\prime}} t} f^{K^{\prime}+}(K, t) \\
& =\left\langle\Psi_{o}\left|A_{K}\right| \Psi_{K^{\prime}}^{+}\right\rangle .
\end{aligned}
$$

We can now use Dyson's equation for $\tilde{\mathscr{G}}$ to derive an effective LippmannSchwinger equation for the time independent scattering amplitude $f^{K^{\prime}+}(K)$. Inserting Dyson's equation (61) into (73) and using (75) leads after some simple manipulations to the equations

$$
\begin{aligned}
f^{K^{\prime}+}(K)= & \lim _{t^{\prime} \rightarrow-\infty} i e^{i \mathscr{E}_{K^{\prime}} t} \tilde{\mathscr{G}}_{K, K^{\prime}}\left(t, t^{\prime}\right) e^{-i \mathscr{E}_{K^{\prime}} t^{\prime}} \\
= & \delta_{K K^{\prime}}+\lim _{t^{\prime} \rightarrow-\infty} i \int d K_{1} d K_{2} d t_{1} d t_{2} \\
& \times e^{i \mathscr{E}_{K^{\prime}} t} \tilde{\mathscr{G}}_{K, K_{1}}^{(p)}\left(t, t_{1}\right) \tilde{\mathscr{T}}_{K_{1}, K_{2}}\left(t_{1}, t_{2}\right) \tilde{\mathscr{G}}_{K_{2}, K^{\prime}}\left(t_{2}, t^{\prime}\right) e^{-i \mathscr{E}_{K^{\prime}} t^{\prime}} .
\end{aligned}
$$


Carrying out the time integrations with the help of property (iii) leads to the desired working equation for the time independent scattering amplitude:

$$
f^{K^{\prime}+}(K)=\delta_{K K^{\prime}}+\int d K_{1} d K_{2} \widetilde{\mathscr{G}}_{K, K_{1}}^{(p)}\left(\mathscr{E}_{K^{\prime}}\right) \tilde{\mathscr{S}}_{K_{1}, K_{2}}\left(\mathscr{E}_{K^{\prime}}\right) f^{K^{\prime}+}\left(K_{2}\right) .
$$

This is an integral equation which can be solved for the scattering amplitude once the self energy $\tilde{\underline{\mathscr{T}}}(\omega)$ is known. It is analogous to the Lippmann-Schwinger equation of basic scattering theory. In the above equation (78) the self energy replaces the potential that appears in the Lippmann-Schwinger equation. Therefore we have actually derived an exact optical model description for our many-body scattering problem: The initial $(N+2)$-particle problem has been reduced to the problem of scattering two particles by an optical potential. The optical potential, which is given by the self energy $\tilde{\mathscr{\mathscr { S }}}(\omega)$, is in general energy-dependent and takes account of all the correlation within the target as well as the interaction between the target and the projectile particles. Like we have seen in Section II E, this self energy becomes energy-independent for systems with particles interacting with one-particle potentials only.

All this is analogous to the elastic scattering of one fermion by a many-body target. In this case the self energy $\underset{\equiv}{\Sigma}(\omega)$ belonging to the single-particle Green's function $\underline{\underline{G}}(\omega)$ takes the role of the optical potential [30,31, 8, 9], which becomes energy independent for systems interacting with a one-particle potential.

\section{E. A Two-Particle Eigenvalue Equation}

In basic scattering theory the Lippmann-Schwinger integral equation presents an alternative formulation to Schrödinger's eigenvalue equation. Thus we can expect that in an optical model description it is, in general, possible to formulate an effective eigenvalue equation for the scattering amplitudes. Multiplying the integral equation (78) with the energy difference $\left(\mathscr{E}_{K^{\prime}}-\mathscr{E}_{K}\right)$ and using the Fourier transform of (62) leads us to the desired equation:

$$
\left(\mathscr{E}_{K}-\mathscr{E}_{K^{\prime}}\right) f^{K^{\prime}+}(K)+\int d K_{2} \tilde{\mathscr{S}}_{K, K_{2}}\left(\mathscr{E}_{K^{\prime}}\right) f^{K^{\prime}+}\left(K_{2}\right)=0
$$

This is an eigenvalue equation in the two-particle Hilbert space. It is formulated using a basis of two-particle wavefunctions that diagonalize the projectile Hamiltonian $\hat{H}_{p}$ defined in III A. Reintroducing $\hat{H}_{p}$ and switching from the quantum number representation to abstract two-particle-space vectors we can rewrite (78) into

$$
\left\{\hat{H}_{p}+\hat{\mathscr{S}}\left(\mathscr{E}_{K^{\prime}}\right)\right\}\left|f^{K^{\prime}}+\right\rangle=\mathscr{E}_{K^{\prime}}\left|f^{K^{\prime}}+\right\rangle .
$$

We have defined the effective scattering kets $\left|f^{K^{\prime}}+\right\rangle$ with the help of the free projectile states $|K\rangle$ of Eq. (51) by

$$
\left\langle K \mid f^{K^{\prime}}+\right\rangle=f^{K^{\prime}+}(K) .
$$


The operator $\hat{\mathscr{S}}\left(\mathscr{E}_{K^{\prime}}\right)$ is defined by its matrix elements:

$$
\left\langle K_{1}|\hat{\mathscr{S}}(\omega)| K_{2}\right\rangle=\tilde{\mathscr{S}}_{K_{1}, K_{2}}(\omega)
$$

Of course, the Green's function $\widetilde{\mathscr{G}}_{K_{1}, K_{2}}(\omega)$ is now rediscovered as a representation of a resolvent $\hat{\mathscr{G}}(\omega)$ that satisfies the following operator identity in two-particle Hilbert space:

$$
\left\{\hat{H}_{p}+\hat{\mathscr{S}}(\omega)-\omega\right\} \hat{\mathscr{G}}(\omega) \equiv \widehat{\mathrm{id}} .
$$

This links up with Section II D, where we already interpreted the extended two-particle propagator as a resolvent of an $\omega$ dependent operator. Now we have finally proven that the scattering propagator (and therefore also the extended two-particle propagator from the last chapter) actually is the resolvent of an effective Hamiltonian that allows to calculate $S$-matrix elements through (80) and (72)! Naturally, the remarks of Section II D on the energy dependence of the effective Hamiltonian apply here in the same way.

We want to remind of the fact that the effective Hamiltonian and therefore also the optical potential derived directly by Feshbach projection contains a divergence that is naturally avoided by our two-particle self energy. To circumvent this divergency in their Feshbach-based approach, Junkin and Villars [21] have to restrict the projectile such that it is made out of unoccupied Hartree Fock orbitals only. They give the effective two-particle scattering potential in terms of an expansion in powers of a projected "residual interaction" that does not allow any scattering between unoccupied orbitals. Our new Green's function optical potential, on the contrary, appears more satisfactory from the conceptual point of view, since no restrictions in the two-particle (projectile) space are necessary. In general, there appears no direct reference to whether a single-particle orbital is occupied or unoccupied in any zeroth order approximation of the ground state. As another important point, the behaviour with respect to one-particle potentials discussed in Section II E provides a contrast between the two approaches. This leads us to the following conclusion: Given some arbitrary two-particle propagator, it is the properties (i), (ii), and (iii) of Section III B that lead to the effective scattering equations of this last chapter. The usefulness of these equations for approximative calculations of scattering parameters, however, is determined by the properties of its self energy. As shown in chapter II, the self energy related to our extended propagator possesses a good-natured energy dependence and behaves well with respect to one-particle potentials. For these reasons the extended two-particle propagator provides a useful tool in scattering. Furthermore it presents an alternative approach that removes drawbacks of the Feshbach projection effective potentials discussed in reference [21], just like the single-particle Green's function does in the case of single-particle projectiles [12]. 


\section{CONCLUSIONS}

In this paper we have presented an extension of the fermionic particle-particle propagator that has algebraic properties similar to the single-particle Green's function. We explicitly constructed one example of an extended propagator and investigated its major algebraic properties. These include an analogon of Dyson's equation with a two-particle self energy. We showed that this self energy serves as an exact optical potential for the scattering of a two-particle projectile by a correlated many-body target. The projectile may either consist of two asymptotically free fermions or a bound two-particle state. In the latter case the two-particle self energy has to be trivially renormalized.

In contrast to effective two-particle potentials derived directly from the multipleparticle Schrödinger equation, the two-particle self energy has a good-natured energy dependence. Additionally, correlations due to a one-particle potential are accounted for exactly already by a first order, energy-independent self energy.

The ideas behind the construction of extended two-particle propagators are quite general and lead to a whole new class of extended two-particle propagators with similar algebraic properties. Employing approximations that aim at ab initio calculations will lead to different working equations for each of these functions. A new feature of the extended propagators is the appearance of degeneracies that may require the use of degenerate techniques necessary when employing perturbation theory based approximations. The reason for this lies in the indefiniteness of the metric used in the construction of the extended propagators. It is an open question, whether or not an extended propagator can be found that combines a definite metric with the advantageous properties we discussed in the present paper.

The present approach can be generalized to construct optical potentials for the scattering of an $M$-particle projectile by a many-body target. The starting point is the introduction of an extended $M$-particle propagator which fulfills a Dyson equation.

\section{REFERENCES}

1. A. L. Fetter and J. D. Walecka, "Quantum Theory of Many-Particle Systems," 1st ed., McGrawHill, New York, 1971.

2. L. S. Cederbaum, W. Domcke, J. Schirmer, and W. von Niessen, Adv. Chem. Phys. 65 (1986), 115.

3. L. S. Cederbaum, Int. J. Quant. Chem. 24 (1990), 393.

4. V. G. Zakrewski and J. v. Ortitz, J. Chem. Phys. 102 (1995), 294.

5. M F. Hermann, K. F. Freed, and D. L. Yeager, Adv. Chem. Phys. 48 (1981), 1.

6. Y. Öhrn and G. Born, Adv. Quant. Chem. 13 (1981), 1.

7. H.-D. Meyer, Phys. Rev. A 40 (1989), 5605.

8. J. S. Bell and E. J. Squires, Phys. Rev. Lett. 3 (1959), 96.

9. M. Namiki, Prog. Theor. Phys. 23 (1960), 629.

10. H. Feshbach, Ann. of Phys. (NY) 5 (1958), 357.

11. H. Feshbach, Ann. of Phys. (NY) 19 (1962), 287. 
12. F. Capuzzi and C. Mahaux, Ann. of Phys. (NY) 245 (1996), 147.

13. J. Schirmer, L. S. Cederbaum, and O. Walter, Phys. Rev. A 28 (1983), 1237.

14. D. A. Pines, "The Many-Body Problem," Benjamin, London, 1962.

15. E. K. U. Gross and E. Runge, "Vielteilchentheorie," 1st ed., Teubner, Stuttgart, 1986.

16. J. Oddershede, P. Jørgensen, and D. L. Yeager, Comp. Phys. Rep. 2 (1984), 33.

17. A. B. Trofimov and J. Schirmer, J. Phys. B 28 (1995), 2299.

18. F. Tarantelli, A. Sgamellotti, and L. S. Cederbaum, Phys. Rev. Lett. 72 (1994), 428.

19. F. Tarantelli and L. S. Cederbaum, Phys. Rev. Lett. 71 (1993), 649.

20. H. Årgen, A. Cesar, and C.-M. Liegener, Adv. Quant. Chem. 23 (1992), 1.

21. W. F. Junkin and F. Villars, Ann. of Phys. (NY) 45 (1967), 93.

22. W. F. Junkin and F. Villars, Ann. of Phys. (NY) 51 (1969), 68.

23. A. Tarantelli and L. S. Cederbaum, Phys. Rev. A 45 (1992), 2790.

24. F. J. Dyson, Phys. Rev. A 75 (1949), 486.

25. F. J. Dyson, Phys. Rev. A 75 (1949), 1736.

26. J. Brand, Diplomarbeit, Ruprecht-Karls-Universität Heidelberg, 1995.

27. F. Mertins and J. Schirmer, Phys. Rev. A 53 (1996), 2140.

28. F. Mertins, J. Schirmer, and A. Tarantelli, Phys. Rev. A 53 (1996), 2153.

29. B Simon, "Quantum Mechanics for Hamiltonians Defined as Quadratic Forms," Princeton University Press, Princeton, New Jersey, 1971.

30. P. Ring and P. Schuck, "The Nuclear Many-Body Problem," Springer, New York, 1980.

31. G. Y. Czanak, H. S. Taylor, and R. Yaris, Adv. Mol. Phys. 7 (1971), 287. 